\title{
Cytological, Biochemical, and Transcriptomic Analyses of a Novel Yellow Leaf Variation in a Paphiopedilum (Orchidaceae) SCBG COP15
}

\author{
Ji Li ${ }^{1,2,+}$, Kunlin Wu ${ }^{1,+}$, Lin Li ${ }^{1}\left(\mathbb{D}\right.$, Meina Wang ${ }^{3}$, Lin Fang ${ }^{1, * \mathbb{D}}$ and Songjun Zeng ${ }^{1,4, *(\mathbb{D})}$ \\ 1 Key Laboratory of South China Agricultural Plant Molecular Analysis and Gene Improvement, \\ South China Botanical Garden, Chinese Academy of Sciences, Guangzhou 510650, China; \\ liji17@scbg.ac.cn (J.L.); wkl8@scbg.ac.cn (K.W.); lilin@scbg.ac.cn (L.L.) \\ 2 University of Chinese Academy of Sciences, Beijing 100049, China \\ 3 Shenzhen Key Laboratory for Orchid Conservation and Utilization/National Orchid Conservation Center of \\ China/Orchid Conservation \& Research Center of Shenzhen, Shenzhen 518114, China; \\ snow-wmn2005@163.com \\ 4 Guangdong Provincial Key Laboratory of Applied Botany, South China Botanical Garden, \\ Chinese Academy of Sciences, Guangzhou 510650, China \\ * Correspondence: linfang@scbg.ac.cn (L.F.); zengsongjun@scib.ac.cn (S.Z.) \\ + These authors contributed equally to this work.
}

Citation: Li, J.; Wu, K.; Li, L.; Wang, M.; Fang, L.; Zeng, S. Cytological, Biochemical, and Transcriptomic Analyses of a Novel Yellow Leaf Variation in a Paphiopedilum (Orchidaceae) SCBG COP15. Genes 2022, 13, 71. https://doi.org/ 10.3390 /genes 13010071

Academic Editor: Serena Aceto

Received: 21 November 2021

Accepted: 23 December 2021

Published: 28 December 2021

Publisher's Note: MDPI stays neutral with regard to jurisdictional claims in published maps and institutional affiliations.

Copyright: (C) 2021 by the authors. Licensee MDPI, Basel, Switzerland. This article is an open access article distributed under the terms and conditions of the Creative Commons Attribution (CC BY) license (https:// creativecommons.org/licenses/by/ $4.0 /)$.

\begin{abstract}
The genus Paphiopedilum, belonging to the Orchidaceae, has high ornamental value. Leaf variations can considerably improve the economic and horticultural value of the orchids. In the study, a yellow leaf mutant of a Paphiopedilum hybrid named P. SCBG COP15 was identified during the in vitro plant culture process; however, little is known about their molecular mechanisms. For this, RNA-seq libraries were created and used for the transcriptomic profiling of $P$. SCBG COP15 and the yellow mutant. The $\mathrm{Chl} a, \mathrm{Chl} b$, and carotenoid contents in the yellow leaves decreased by approximately $75.99 \%, 76.92 \%$, and $56.83 \%$, respectively, relative to the green leaves. Decreased chloroplasts per cell and abnormal chloroplast ultrastructure were observed by electron microscopic investigation in yellowing leaves; photosynthetic characteristics and $\mathrm{Chl}$ fluorescence parameters were also decreased in the mutant. Altogether, 34,492 unigenes were annotated by BLASTX; 1,835 DEGs were identified, consisting of 697 upregulated and 1138 downregulated DEGs. HEMA, CRD, $C A O$, and CHLE, involved in Chl biosynthesis, were predicted to be key genes responsible for leaf yellow coloration. Our findings provide an essential genetic resource for understanding the molecular mechanism of leaf color variation and breeding new varieties of Paphiopedilum with increased horticultural value.
\end{abstract}

Keywords: Paphiopedilum hybrid; leaf variegation; chlorophyll metabolism; transcriptome analysis

\section{Introduction}

Leaves are vital organs in plants for photosynthesis, respiration, and nutrient transformation, as well as an important aesthetic trait in ornamental plants. Commonly, chlorophyll (Chl), carotenoids, and anthocyanins are the main leaf pigments that are determined by genotype and growth environment [1]. Chl is the primary photosynthetic pigment found in leaves. It is able to capture light energy and transfer it to the photoreaction center to generate chemical energy during photosynthesis [2]. Recently, most research has focused on red leaves, which are associated with anthocyanin accumulation [3]. However, the yellowing of leaves has received significantly less attention, and most studies on leaf yellowing have focused on Chl metabolism [4-6].

In Arabidopsis thaliana, a total of 27 genes participating in $\mathrm{Chl}$ biosynthesis have been identified (Supplementary Figure S1), including those contributing to Glu-tRNA, Chl $a$, and $\mathrm{Chl} b$ production [2], and mutations at any position may lead to low Chl content, resulting 
in abnormal leaf coloration [7]. For instance, the RNA silencing of HEMA in barley caused varying degrees of inhibition of $\mathrm{Chl}$ biosynthesis, and as a result the plant showed an albino and yellow phenotype [8]. In rice, mutations in the CHLI and CHLD genes lead to a decrease in enzyme activity and $\mathrm{Chl}$ content [9]. Chl degradation is also one of the main reasons for leaf discoloration. Generally, the biosynthesis and degradation of $\mathrm{Chl}$ are in dynamic equilibrium within the metabolic process, which is essential for green plants; once the Chl degradation pathway related genes are abnormal, the plant will show the corresponding abnormal leaf color [10]. In rice, NYC1 and NOL can form a complex and act as a $\mathrm{Chl} b$ reductase to catalyze Chl degradation [11]. The high expression of NYE1 in A. thaliana causes yellowing and even albino leaves, and it was confirmed that NYE1 intervenes with the regulation of PAO activity during the Chl degradation process [12].

Paphiopedilum, also known as the Venus slipper orchid and Cinderella, has a high ornamental value due to its unique flower type, gorgeous flower color, long-lasting flowering period, and elegant, upright leaves. It has long become a very popular upscale flower in the international flower market [13]. In orchids, leaf variations such as striped leaves, yellow leaves, and spotted leaves have recently gained increased popularity among breeders and customers. Accordingly, these extraordinary traits significantly improve the economic and horticultural value of the plants [14-18]. Presently, large-scale production of Paphiopedilum seedlings is mainly performed through tissue culture [19]. A Paphiopedilum hybrid variety named $P$. SCBG COP15 has a small probability of developing golden leaves during its tissue culture process; however, the understanding of the underlying molecular mechanisms in the yellow leaf mutation remains limited. This phenotype is stably inherited; therefore, we obtained this yellow leaf mutant of $P$. SCBG COP15 for further investigation.

In this research paper, photosynthetic pigments and chloroplast ultrastructure, together with transcriptomics, were compared between yellow mutant leaves and the green normal leaves of $P$. SCBG COP15. We identified differentially expressed genes (DEGs) and transcription factors (TFs) related to Chl metabolism, and evaluated the expression levels of some key unigenes by quantitative real-time polymerase chain reaction (qRT-PCR) to validate their involvement in leaf variation. Our findings illustrate the physiological, cytological, and bioinformatic aspects of yellow leaf mutants of P. SCBG COP15, and they lay the foundation for further understanding of yellow leaf coloration and the facilitation of molecular breeding in Paphiopedilum.

\section{Materials and Methods}

\subsection{Plant Material and Sample Preparation}

One-year-old seedlings of the Paphiopedilum hybrid named P. SCBG COP15, along with the yellow leaf variety which formed through the tissue culture by using lateral bud explants were used in present study. P. SCBG COP15 was a combination between P. Maudiae as female parent and P. SCBG Yunzhijun as male parent, The experimental materials were grown in the tissue culture laboratory and greenhouse of the South China Botanical Garden, Chinese Academy of Sciences, Guangzhou, China (Supplementary Table S1). Fresh and mature leaves were collected for physiological observation, determination of photosynthetic characteristics, and detection of $\mathrm{Chl}$ fluorescence parameters. The samples were stored at $-80^{\circ} \mathrm{C}$ for biochemical experiments and RNA extraction.

\subsection{Pigment Content Measurement}

Approximately $0.1 \mathrm{~g}$ fresh leaf tissue was extracted with $80 \%$ acetone overnight at $4{ }^{\circ} \mathrm{C}$, and the absorbance was measured at $646.8 \mathrm{~nm}, 663.2 \mathrm{~nm}$, and $470 \mathrm{~nm}$ in a 96-well plate with a microplate reader (Tecan Infinite, Männedorf, Switzerland). The $\mathrm{Chl}$ and carotenoid content was calculated according to Lichtenthaler [20].

\subsection{Photosynthetic Characteristics and Chlorophyll Fluorescence Parameter Measurement}

An LI-6800 portable photosynthesis system (Li-cor, Lincoln, NE, USA) was used to measure photosynthetic characteristic parameters, including intercellular $\mathrm{CO}_{2}$ concentra- 
tion (Ci), stomatal conductance (Gs), and net photosynthetic rate (Pn). The fluorescence parameters were measured using a Chl fluorescence spectrometer (Heinz Walz $\mathrm{GmbH}$, Effeltrich, Germany), with the leaves left in the dark for approximately $30 \mathrm{~min}$. Finally, $\mathrm{F}_{\mathrm{o}}$, $F_{m}, N P Q, q N$, and $Y(I I)$, along with $F_{v} / F_{m}$, were measured.

\subsection{Anatomy Observations}

Leaf samples of the normal wild type of $P$. SCBG COP15 (G), and its yellow mutant $(\mathrm{Y})$, were cut into approximately $1 \mathrm{~mm} \times 2 \mathrm{~mm}$ pieces and were fixed with $0.1 \mathrm{M}$ phosphate buffer $(\mathrm{pH}=7.2)$, containing $2.5 \%$ glutaraldehyde and $2 \%$ paraformaldehyde. After washing the leaf samples six times with the $0.1 \mathrm{M}$ phosphate buffer, the samples were postfixed in $1 \%$ osmium tetroxide for $4 \mathrm{~h}$ and subsequently washed again with $0.1 \mathrm{M}$ phosphate buffer. Ultrathin sections $(80 \mathrm{~nm}$ ) were cut using an ultramicrotome (Leica UC7, Leica Microsystems, Wetzlar, Germany), which were then stained with $4 \%$ uranyl acetate and $2 \%$ lead citrate. Ultrathin sections of the leaf ultrastructures were observed using transmission electron microscopy (TEM) (transmission electron microscope JEOL JEM-1010, Tokyo, Japan), operating at $100 \mathrm{kV}$. For the characterization of the leaf architecture, fresh leaf samples from $\mathrm{G}$ and $\mathrm{Y}$ were first fixed in $4 \%$ agar, and then cut into $200 \mu \mathrm{m}$ tissue slices using a vibratome (Leica VT 1200, Leica Microsystems, Wetzlar, Germany) at $1.00 \mathrm{~mm} / \mathrm{s}$ speed and $3.00 \mathrm{~mm}$ amplitude. The slices were observed under a Leica DVM6 ultra-depth field microscope (Leica Microsystems, Wetzlar, Germany).

\subsection{RNA Extraction, Library Construction, Sequencing}

The leaf samples were ground into a fine powder in liquid nitrogen. Total RNA was isolated using an RNA kit (Polysaccharides \& Polyphenolics-rich) (Hua Yueyang, Beijing, China), with the use of RNase-free DNase I (Takara Bio, Shiga, Japan) to remove genomic DNA contamination. The integrity and purity of the total RNA was determined using a 2100 Bioanalyzer (Agilent Technologies, Inc., Santa Clara, CA, USA) and quantified using the ND-2000 NanoDrop (Thermo Scientific, Wilmington, DE, USA). Only high-quality RNA samples were used to prepare RNA-seq libraries. Independent quadruplication of whole-leaf materials from $P$. SCBG COP15 and its yellow leaf variety were used to construct the libraries and sequences with a Illumina TruSeqTM RNA sample preparation Kit (San Diego, CA, USA) and the Illumina HiSeq ${ }^{\mathrm{TM}} 6000$ sequencing platform (Illumina, San Diego, CA, USA) by Shanghai Majorbio Bio-pharm Biotechnology Co., Ltd. (Shanghai, China), respectively.

\subsection{Transcriptome Assembly and Annotation}

The total clean reads from the eight libraries were assembled de novo using Trinity software (http:/ / trinityrnaseq.sourceforge.net/ 10 November 2021), with the use of the default parameters. The assembled unigenes were searched against the public databases, including the NCBI non-redundant protein (NR) database (http:/ / www.ncbi.nlm.nih.gov), SwissProt (http:/ /www.expasy.ch/sprot/), Clusters of Orthologous Groups (COG) database (http:/ / www.ncbi.nlm.nih.gov/COG), Gene Ontology (GO) functional annotation (http: //www.blast2go.com/b2ghome) [21], and the Kyoto Encyclopedia of Genes and Genomes (KEGG) database [22].

\subsection{DEGs Identification and Functional Enrichment Analysis}

DEGs were identified using DESeq2 [23] and unigenes. A fold change (FC) $>2$ or $<-2$ and an adjusted $p$-value $\leq 0.05$, considered the transcripts per million reads (TPM) significantly differentially expressed. All DEGs were then mapped to GO terms and KEGG pathways in the respective databases using Goatools (https://github.com/tanghaibao/ Goatools) and KOBAS (http://kobas.cbi.pku.edu.cn/home.do) [24]. 


\subsection{Validation of DEGs by $q R T-P C R$}

A total of $1 \mu \mathrm{g}$ of high quality total RNA (the same sample used in RNA-Seq) was reverse-transcribed to first-strand cDNA using TransScript ${ }^{\circledR}$ One-Step gDNA Removal cDNA Synthesis SuperMix (Transgen, Beijing, China) to perform qRT-PCR. Twelve unigenes related to $\mathrm{Chl}$ biosynthesis and degradation were selected for further validation. Specific primers were designed with the Primer Premier software (version 5.0), and qRT-PCR was performed in a 384-well block with PerfectStart Green qPCR SuperMix (Transgen, Beijing, China) on a LightCycler 480II (Roche, Mannheim, Germany). The reaction conditions were set as follows: $95{ }^{\circ} \mathrm{C}$ for $30 \mathrm{~s}, 40$ cycles at $94{ }^{\circ} \mathrm{C}$ for $5 \mathrm{~s}$, and $60{ }^{\circ} \mathrm{C}$ for $30 \mathrm{~s}$. Cq values were analyzed using the LightCycler ${ }^{\circledR} 480$ software. The relative unigene expression levels of target genes were determined by the $2^{-\Delta \Delta C T}$ method [25], with the actin gene (TRINITY_DN13664_c0_g3) as the internal control.

\section{Results}

\subsection{Leaf Anatomical Characteristics and Ultrastructure}

As shown in Figure 1D, Chl was present throughout the whole mesophyll cell, and no pigment was observed in the leaf epidermis in neither the green nor yellow leaves. The mutant leaves showed a light-yellow color compared to the green color in the normal leaves. Furthermore, we found that chloroplasts showed the typical structures of green leaf mesophyll cells, with normal grana and thylakoids present. However, only a few thylakoids remained in the chloroplasts of yellow mutants and showed damaged thylakoid membranes, absent stromal lamellae, and a large number of irregularly arranged round vesicles (Figure 2). Additionally, the average number of chloroplasts in each cell in normal green leaves was 1.89-fold higher than that in yellow mutant leaves (Figure 3B). This result indicates that the variation in the leaf color of the yellow mutant might be a consequence of damage from chloroplast development.

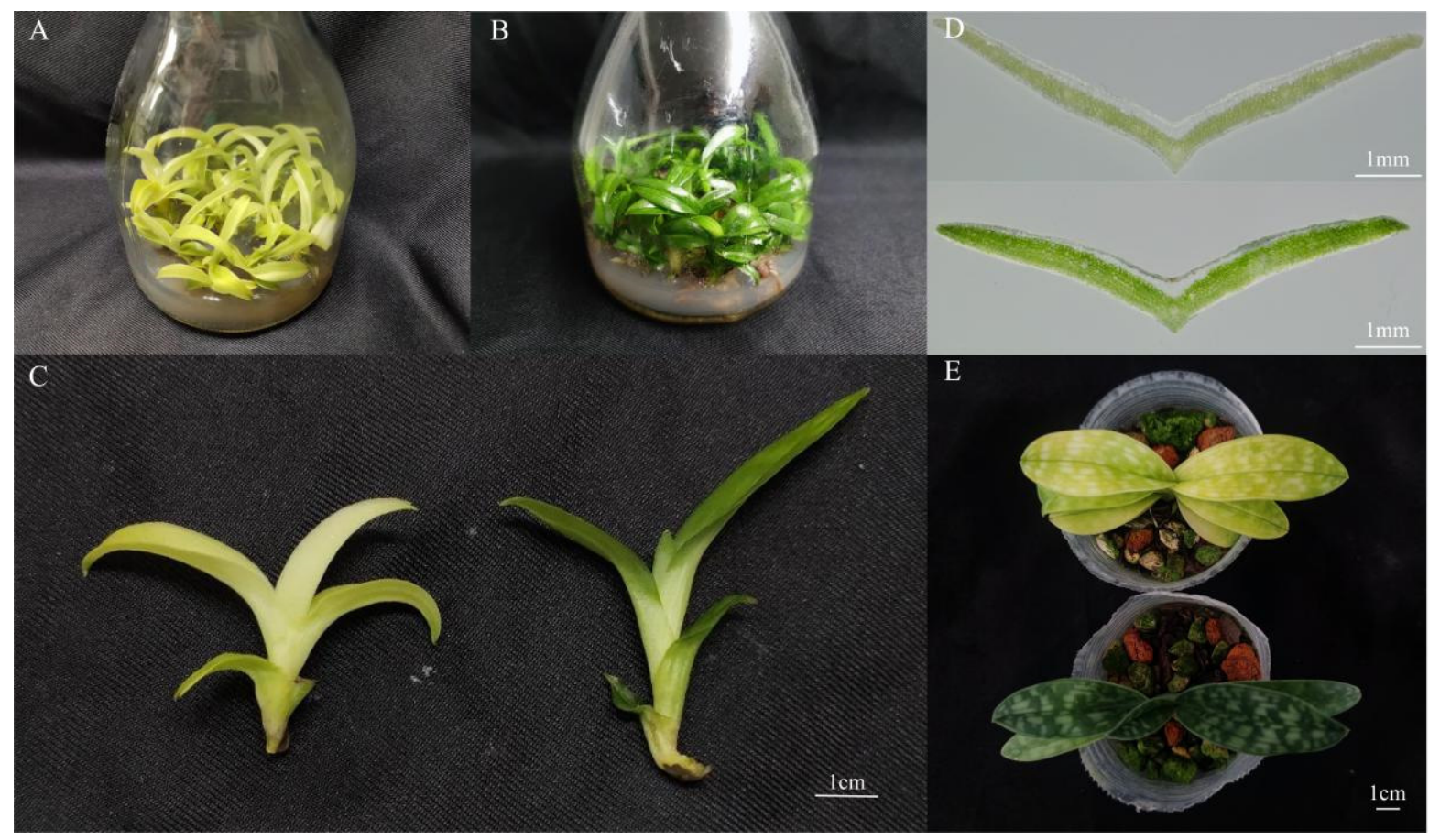

Figure 1. Phenotype of $P$. SCBG COP15 and the yellow mutant. (A) Phenotype of $P$. SCBG COP15. (B) Phenotype of the yellow mutant. (C) One year old seedling of P. SCBG COP15 (right) compared with the yellow leaf variety (left). (D) The anatomical distribution of pigments in P. SCBG COP15 (below) and the yellow mutant leaves (above). (E) Seedlings of the P. SCBG COP15 (below) and the yellow leaf variety (above) growing in the Orchid greenhouse. 


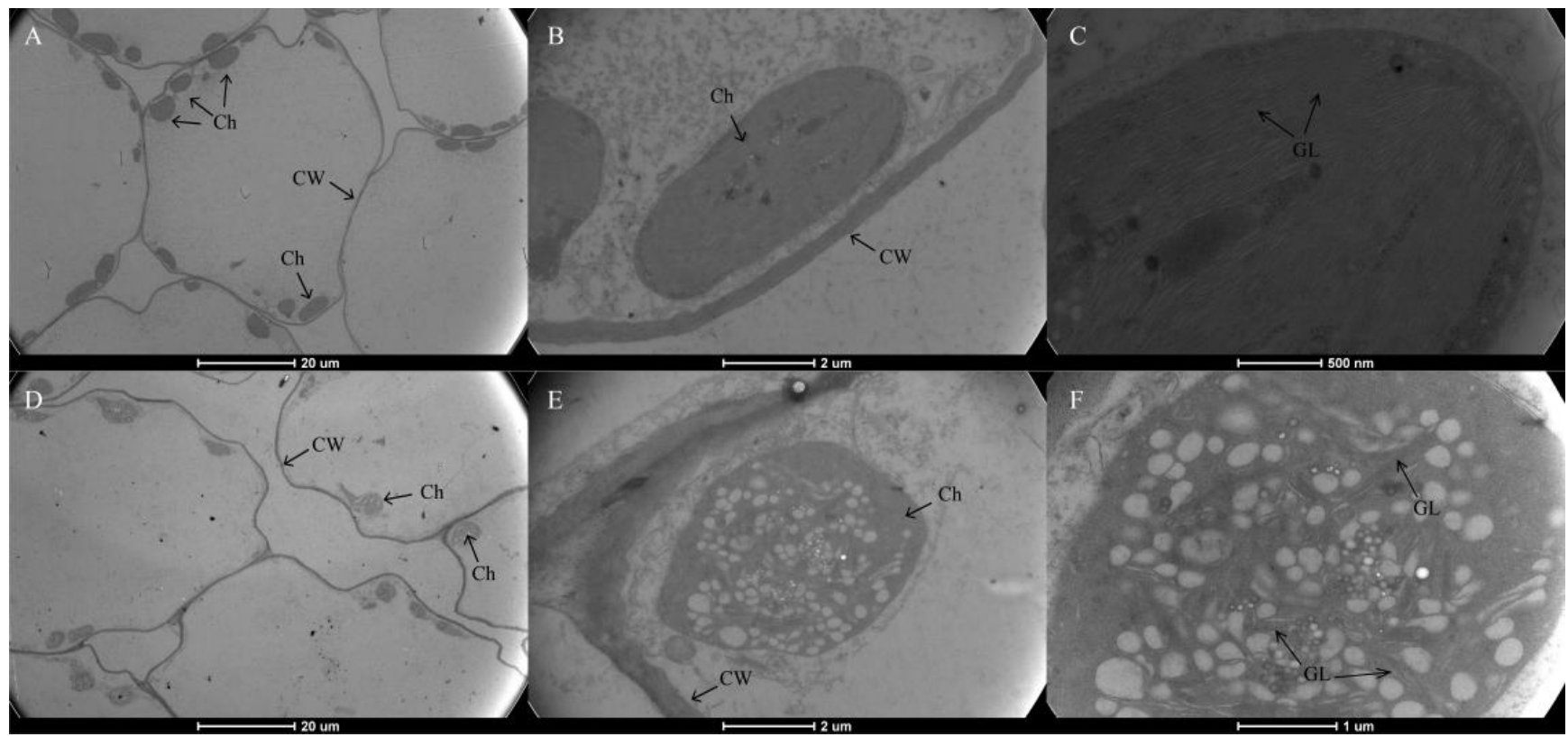

Figure 2. Transmission electron micrograph of chloroplasts from P. SCBG COP15 and the yellow mutant. (A-C) Chloroplast ultrastructures in the green leaves were intact, clearly visible, and wellorganized. (D-F) The ultrastructure of mutant chloroplasts had serious defects, were packed with vesicles, and are filled with many plastid spheres. Ch: chloroplast; CW: Cell Wall; GL: grana lamella.
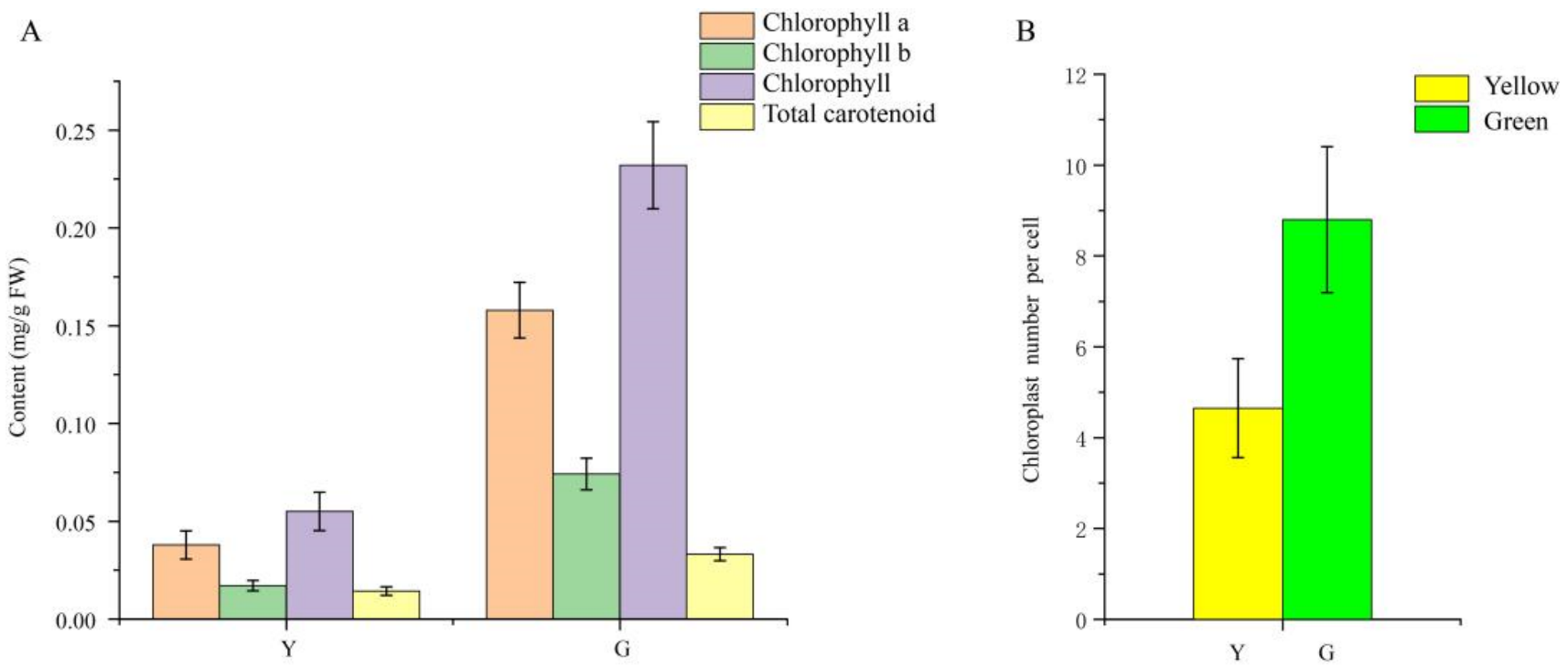

Figure 3. Pigment accumulation and the average number of chloroplasts per cell in $P$. SCBG COP15 and the yellow mutant. (A) Chlorophyll a, chlorophyll b, chlorophyll, and total carotenoid contents between $Y$ (yellow mutant) and G (green normal leaves). (B) The average number of chloroplasts per cell decreased by $47.16 \%$ in the mutant leaves.

\subsection{Photosynthetic Characteristics and Chlorophyll Fluorescence Parameters}

Six Chl fluorescence parameters were ascertained in the present study, and it was obvious that all parameters of the normal green leaf were higher than those of the yellow mutant; specifically, the $\mathrm{F}_{\mathrm{v}} / \mathrm{F}_{\mathrm{m}}$ value of yellow leaves was only $41.7 \%$ of the green leaves value (Figure 4), indicating that light absorption and energy transfer of the light-harvesting complexes were more efficient in the normal green leaf than in the yellow mutant. Next, the photosynthetic characteristic parameters, including intercellular $\mathrm{CO}_{2}$ concentration $(\mathrm{Ci})$, stomatal conductance (Gs), and net photosynthetic rate (Pn), were determined for further 
analysis. The $\mathrm{Ci}, \mathrm{Gs}$, and Pn values of the green leaves were approximately $35.93,73.36$, and $60.24 \%$ higher than those of the yellow leaf (Figure 4B-D), respectively, suggesting a stronger photosynthetic capacity in green leaves compared to the mutant.

A

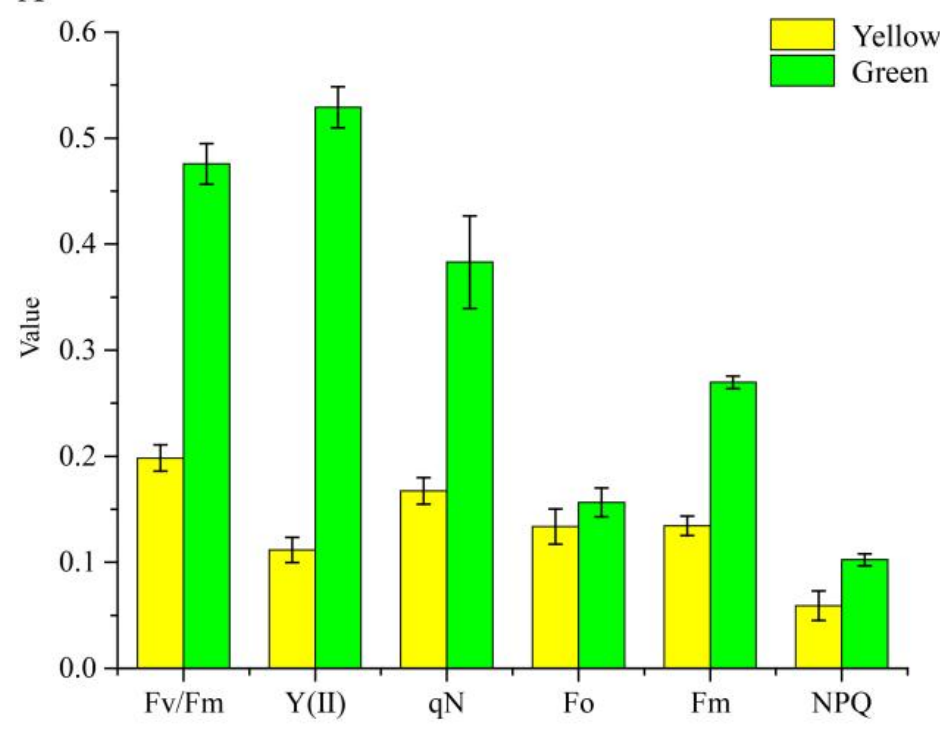

$\mathrm{C}$

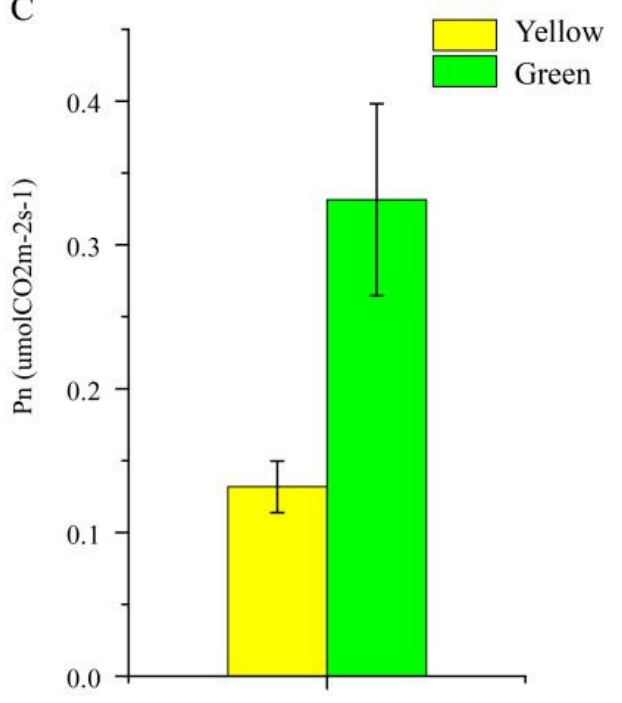

B

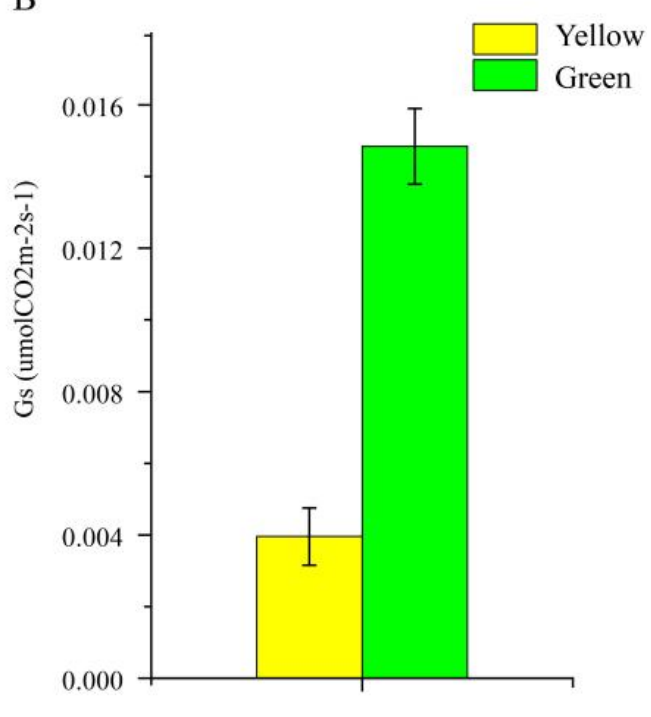

D

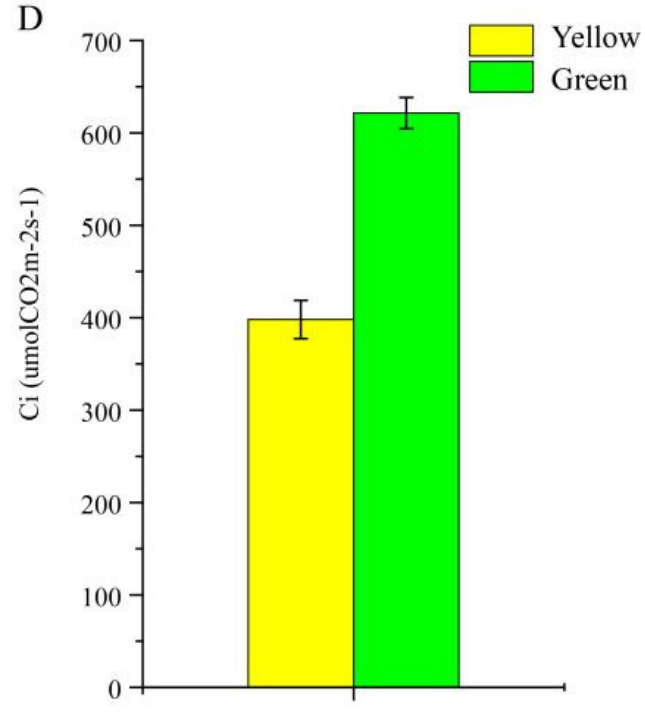

Figure 4. Photosynthetic characteristics (A) and three chlorophyll fluorescence parameters (B-D) of $P$. SCBG COP15 and the yellow mutant.

\subsection{Library Construction and De Novo Assembly}

To reveal the molecular mechanism of the yellow-leaf phenotype in P. SCBG COP15, high-quality RNA samples extracted from the leaves of $G$ and $Y$ were applied to construct the sequencing library with four biological replicates (Supplementary Figures S2 and S3). The number of clean reads for each library ranged from 40,802,964 to $53,349,426$, with a mapped ratio of $67.95 \%$ to $71.91 \%$. A total of 79,129 unigenes were identified from the mapped libraries, as well as 113,567 transcripts obtained, with an N50 length of $1590 \mathrm{bp}$. The GC content ranged from $47.77 \%$ to $49.43 \%$, and the Q30 base percentage was $\geq 94.71 \%$, which indicated a high read confidence level (Supplementary Tables S2-S4). 


\subsection{Functional Annotation and Classification}

In total, 34,492 unigenes were annotated in the NR, Nt, SwissProt, KEGG, COG, Pfam, and GO databases using BLASTX, which accounted for $43.59 \%$ of the assembled unigenes. In the NR annotated species distribution, the top three matched species were Dendrobium catenatum, Phalaenopsis equestris, and Apostasia shenzhenica (Supplementary Figure S4). Of these annotated unigenes, 28,116 (35.53\%) were categorized as contributing to a biological process, cellular component, or molecular function in the GO database. Binding, catalytic activity, and cellular processes were the three prominent subclasses. A total of 9364 unigenes were annotated in the KEGG functional classification, and unigenes accounted for the highest percentage of translation, carbohydrate biosynthesis and folding, sorting, and degradation (Supplementary Figures S5 and S6).

\subsection{DEGs Analysis and Verification}

DEGs of $Y$ and $G$ were analyzed based on the TPM values of the unigenes. A total of 1835 DEGs were identified ( $P$ adj. $\leq 0.05, F C \geq 2$ ) by pairwise comparison, with 697 upregulated and 1138 downregulated DEGs observed. To obtain a functional categorization of the DEGs, they were annotated for GO analysis. Next, 1104 DEGs were categorized in three main GO classification categories, among which polysaccharide metabolic process, polysaccharide catabolic process, and extracellular region were the three most frequently identified terms (Figure 5). In addition, KEGG annotation was performed to identify the specific biochemical pathways involved in leaf variegation, with 463 DEGs successfully assigned to 111 KEGG pathways. The top 20 enriched KEGG pathways are displayed in Figure 6, and the most frequently represented pathways were plant hormone signal transduction, phenylpropanoid biosynthesis, and starch and sucrose metabolism, with 29, 21, and 17 DEGs observed, indicating that plant hormones appear to be playing an important role in the formation of plants leaf yellowing.

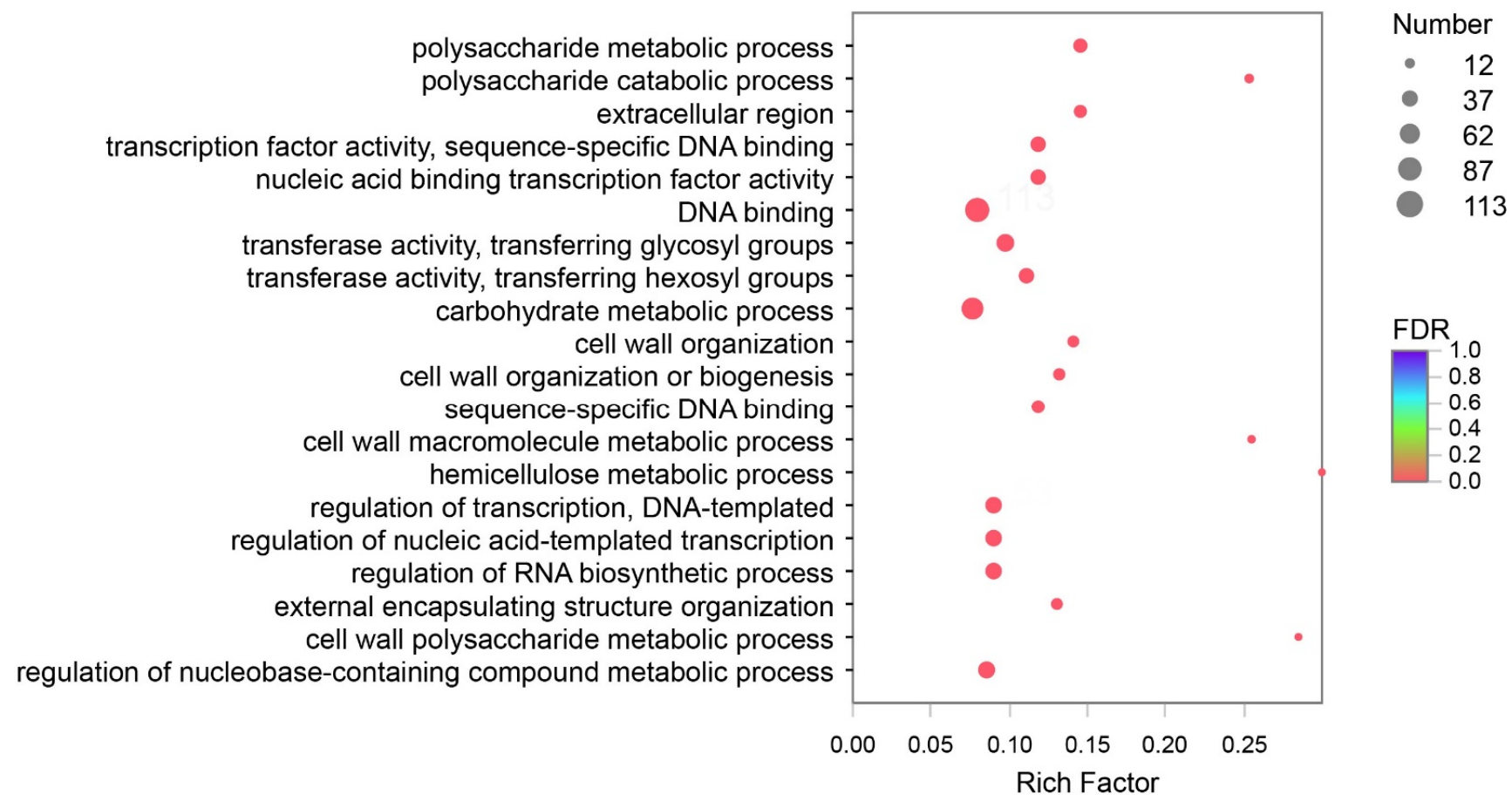

Figure 5. Gene Ontology (GO) enrichment analysis with the differentially expressed genes (DEGs). A total of 1104 DEGs were categorized in three main GO classification categories. 


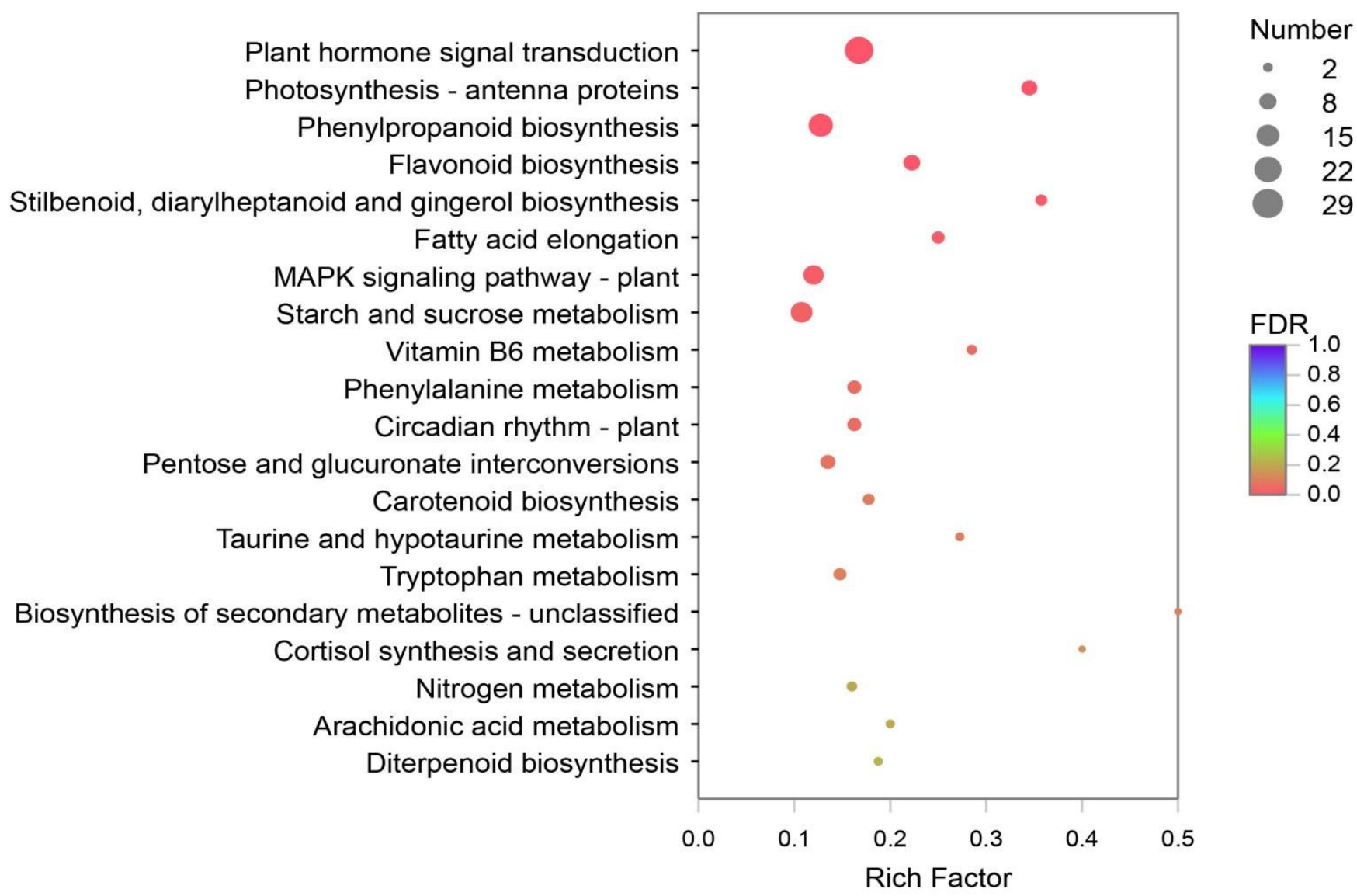

Figure 6. The Kyoto Encyclopedia of Genes and Genomes (KEGG) enrichment analysis with the DEGs. A total of 463 DEGs were assigned to 111 KEGG pathways, and the plant hormone signal transduction pathways were most frequently represented.

\subsection{Expression Pattern of the Genes Involved in Chlorophyll Biosynthesis and Degradation}

In the present study, 15 unigenes related to $C h l$ biosynthesis and 11 unigenes associated with Chl degradation were identified based on RNA-seq annotation. HEMA(TRINITY_DN 6358_c0_g1), the first structural gene in the Chl biosynthesis pathway, was significantly downregulated in the mutant, indicating that its decreased expression might contribute to the low Chl biosynthetic efficiency of Glutamate-1-semialdehyde (GSA). Furthermore, the protein and enzymatic chloroplastic precursor coding genes CRD (TRINITY_ DN2556_c0_ g1) and CAO (TRINITY_DN1555_c0_g1), respectively, also showed low expression in the yellow leaves. In contrast, the Chl degradation genes, especially NYC1-1 (TRINITY_DN221_c0_g1) and PPH3 (TRINITY_DN3203_c0_g1), showed significantly increased mRNA levels in the yellow leaves (Figure 7). This result indicates that the downregulation of key Chl biosynthesis genes, along with the upregulation of Chl degradation genes, may directly cause the decrease of $\mathrm{Chl}$ and consequently lead to the yellow leaf phenotype. We also identified that a unigene for the probable transcription factor GLK1 (ID: TRINITY_DN5163_c0_g1, D. catenatum) was upregulated 2.11-fold in the normal green leaves compared with the mutant, which also indicates the important role of GLK (Golden 2-like) in leaf coloration. Moreover, the GLK gene family acts as a vital transcription factor to regulate chloroplast development, leaf color, hormone signal transduction, biological and abiotic stress and plant senescence, etc [26-28]. CDS and protein sequences that participated in Chl biosynthesis and degradation were screened from RNA-Seq data and cloned with the assistance of PCR to confirm the accuracy of the analysis and for further study (Supplementary Table S5). 
A

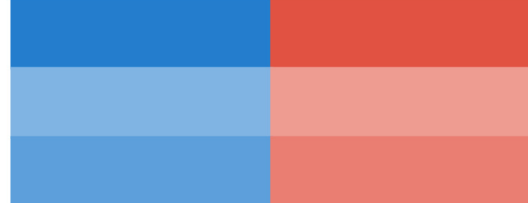

$\begin{array}{lll}\text { TRINITY_DN6358_c0_g1 } & \text { HEMA } & -0.2 \\ \text { TRINITY_DN2556_c0_g1 } & \text { CRD } & -0.1 \\ \text { TRINITY_DN1555_c0_g1 } & \text { CAO } & -0.1 \\ & & -0.2\end{array}$

TRINITY_DN10827_c0_g2 CHLH

TRINITY_DN6654_c0_g1 HEMB

TRINITY_DN6221_c0_g1 PORA/B/C-1

TRINITY_DN25261_c0_g3 HEMC

TRINITY_DN4989_c0_g2 HEME2

TRINITY_DN6865_c0_g1 HEMD

TRINITY_DN1733_c0_g1 PORA/B/C-2

TRINITY_DN26992_c0_g2 HEME1

TRINITY_DN1919_c0_g1 DVR

TRINITY_DN11386_c0_g2 CHLG

TRINITY_DN8458_c0_g1 HEML

TRINITY_DN906_c0_g1 HEMF

Yellow leaves Green leaves

B

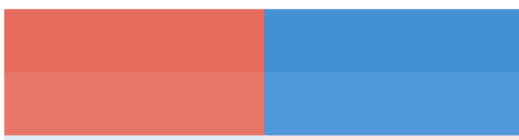

TRINITY_DN3203

TRINITY_DN221_c0_g

TRINITY_DN3768_c0_g1

TRINITY_DN2611_c0_g1

TRINITY DN2096 c0 g2 $\quad \mathrm{PaO} 1$

TRINITY_DN86_c0_g1 PPH4

TRINITY_DN9289_c0_g1 $\mathrm{PaO3}$

TRINITY_DN482_c0_g1 HCAR

TRINITY DN38062 c0 g1 $\mathrm{PaO} 2$

TRINITY_DN16260_c0_g1 PPH1

TRINITY_DN1378_c0_g1 RCCR

Yellow leaves Green leaves

Figure 7. Expression profiles of unigenes involved in chlorophyll biosynthesis and degradation. The computing method of the expression profiles: The logarithm value of unigene TPM in each sample was first taken, and then hierarchical clustering is used for standardization. (A) 15 unigenes related to $\mathrm{Chl}$ biosynthesis. (B) 11 unigenes associated with Chl degradation. 


\subsection{TFs Involved in Leaf Coloration}

TFs regulate the complex transcription network and therefore play a crucial role in regulating gene expression in a series of plant biological processes. In the present study, we identified 102 differentially expressed TFs belonging to 25 TF families, and the top five abundant TF families were the MYB superfamily $(16,15.6 \%)$, NAC (16, 15.6\%), C2C2 (12, $11.7 \%)$, bHLH $(10,9.8 \%)$, and AP2/EPF (7, 6.8\%). We analyzed 20 DEGs that exhibited highly significant downregulation or upregulation between yellow and green leaves and found that bHLH TFs were downregulated in the yellow mutant leaves, while most MYB superfamily TFs showed an upregulation trend in the mutant compared to normal green leaves. This suggests the TF's vital function in leaf coloration (Figure 8; Supplementary Table S6).

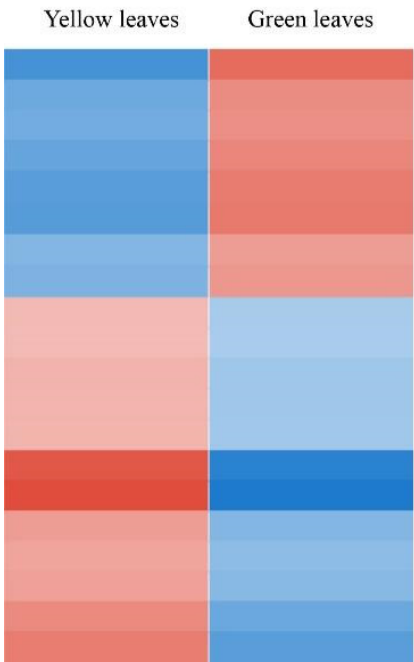

\begin{tabular}{ll}
\multicolumn{1}{c}{ Unigene ID } & \multicolumn{1}{c}{ TF families } \\
TRINITY_DN9180_c0_g1 & bHLH \\
TRINITY_DN6406_c0_g1 & bHLH \\
TRINITY_DN79390_c0_g1 & bHLH \\
TRINITY_DN4589_c0_g2 & AP2/ERF \\
TRINITY_DN4153_c0_g2 & bHLH \\
TRINITY_DN21810_c0_g2 & AP2/ERF \\
TRINITY_DN72300_c0_g1 & MYB_superfamily \\
TRINITY_DN9373_c0_g1 & bHLH \\
TRINITY_DN4294_c0_g1 & MYB_superfamily \\
TRINITY_DN72553_c0_g1 & C2C2 \\
TRINITY_DN1508_c0_gl & NAC \\
TRINITY_DN4938_c0_g1 & C2C2 \\
TRINITY_DN6605_c0_g1 & C2C2 \\
TRINITY_DN5451_c0_g1 & MYB_superfamily \\
TRINITY_DN4362_c0_g1 & MYB_superfamily \\
TRINITY_DN42864_c0_g1 & MYB_superfamily \\
TRINITY_DN8429_c0_g1 & MYB_superfamily \\
TRINITY_DN16816_c0_g1 & NAC \\
TRINITY_DN10166_c0_g1 & AP2/ERF \\
TRINITY_DN41955_c0_g1 & NAC
\end{tabular}

Description
transcription factor HEC2 [Dendrobium catenatum]
transcription factor PIF4 isoform X2 [Dendrobium catenatum]
putative transcription factor bHLH086 [Dendrobium catenatum]
ethylene-responsive transcription factor ERF039-like [Phoenix dactylifera]
transcription factor PIL13 isoform X1 [Dendrobium catenatum]
ethylene-responsive transcription factor ERF113 [Dendrobium catenatum]
hypothetical protein Ahy_A03g015667 [Arachis hypogaea]
transcription factor PIF3-like isoform X1 [Dendrobium catenatum]
transcription factor GAMYB-like isoform X2 [Phoenix dactylifera]
cyclic dof factor 4-like [Dendrobium catenatum]
NAC domain-containing protein 48-like [Musa acuminata subsp. malaccensis]
putative GATA transcription factor 22 [Dendrobium catenatum]
GATA transcription factor 9-like isoform X2 [Dendrobium catenatum]
myb-related protein MYBAS2-like [Phalaenopsis equestris]
transcription factor MYB12 [Dendrobium catenatum]
transcription factor NIGT1 [Dendrobium catenatum]
myb-related protein MYBAS1-like [Dendrobium catenatum]
NAC domain-containing protein 2 [Dendrobium catenatum]
ethylene-responsive transcription factor ERF110 [Dendrobium catenatum]
NAC domain-containing protein 2 [Dendrobium catenatum]

Figure 8. DEGs analysis of transcription factors (TFs) in normal green leaves and mutant leaves. Expression profiles and RNA-seq description of 20 DEGs which exhibited highly significant down or upregulation between yellow and green leaves.

\subsection{Validation of Gene Expression Profiling}

Twelve candidate unigenes involved in $\mathrm{Chl}$ biosynthesis and degradation were selected to test the validity of the transcriptomic data using qRT-PCR analysis. HEMA, CHLE and CAO participated in Chl biosynthesis, their genes showing a significant high expression level in green leaves, while Chl degradation genes including NYC1-1, HCAR, PPH1, PPH2, and RCCR were dramatically downregulated in green leaves. The results showed that the expression patterns revealed by qRT-PCR analysis were consistent with the transcriptomic data (Figure 9). Primers used in qRT-PCR was provided in Supplementary Table S7. 

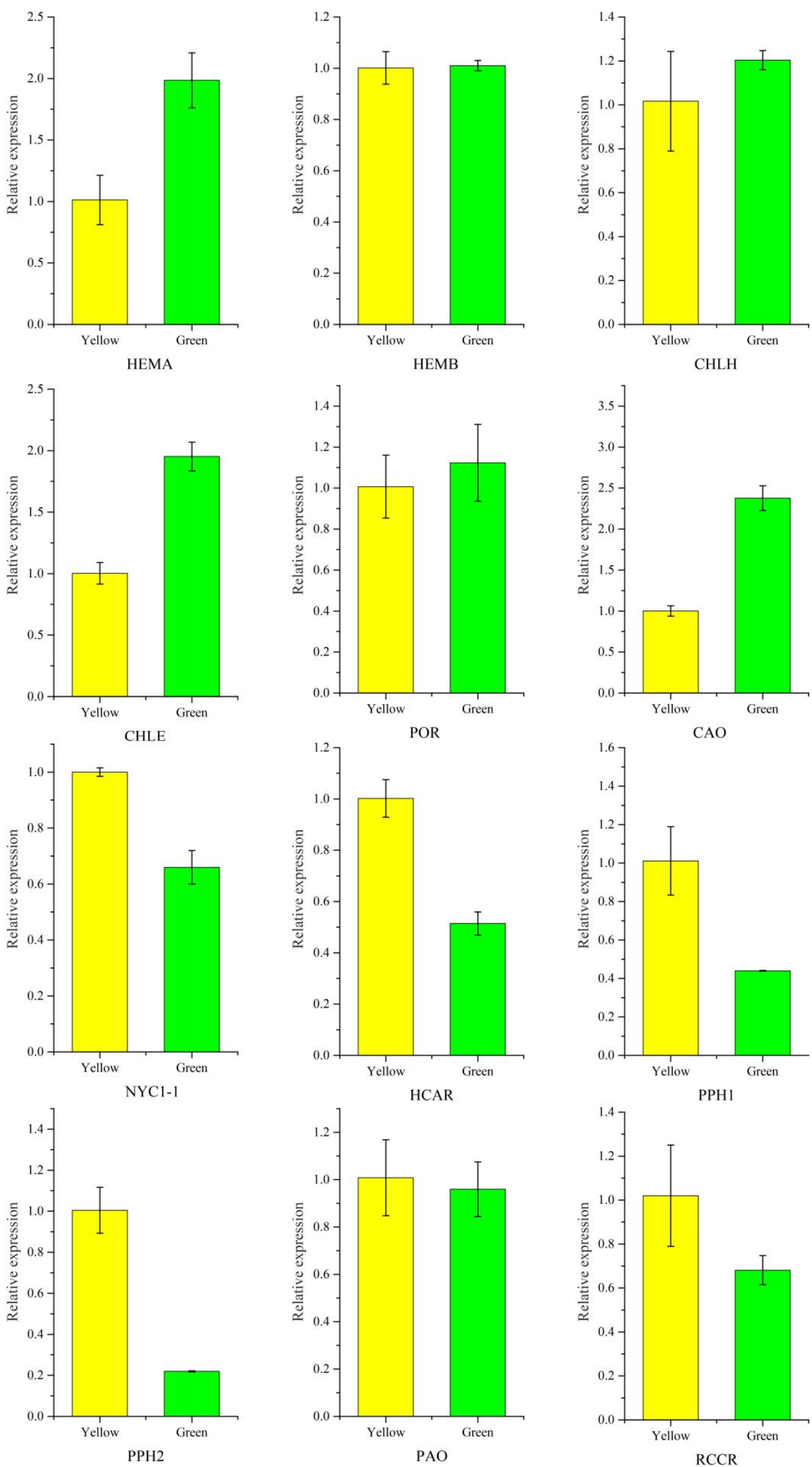

Figure 9. Validation of chlorophyll-related gene expression between P. SCBG COP15 and the yellow mutant. Expression levels were calculated by the $2^{-\Delta \Delta C T}$ method with the actin gene (TRINITY_DN13664_c0_g3) as the internal control. 


\section{Discussion}

Paphiopedilum is one of the most fashionable and rare orchid genera because of its high ornamental value, while wild populations of Paphiopedilum are facing the threat of extinction due to excessive collection and habitat destruction [29]. Presently, large-scale in vitro propagation is the main option for producing Paphiopedilum seedlings [30]. It is a common phenomenon that plant somatic cells undergo somaclonal variation during tissue culture. Therefore, this method is usually used to induce and obtain mutants in the process of plant breeding [31], which has been reported in wheat [32], the Chrysanthemum genus [33], Saintpaulia [34], and the Cymbidium genus [35]. In the present study, the yellow leaf mutant along with $P$. SCBG COP15, the green normal leaf hybrid discovered during the tissue culture process, were used as the materials to study leaf color variation in Paphiopedilum. Our results not only identified candidate structural and regulatory genes involved in leaf coloration, but also provided insight into golden leaf formation in Paphiopedilum.

$\mathrm{Chl}$ is the main pigment that harvests solar energy in leaf tissues and makes the leaves appear green. In the present study, the photosynthetic pigment content, including $\mathrm{Chl}$ $a, \mathrm{Chl} b$, and carotenoid, in the green normal leaves was significantly higher than that of the mutant. Many Chl deficient mutants were reported in maize [36] and A. thaliana [37]. Generally, thylakoid membranes, arranged regularly and stacked into grana in the chloroplasts, are vital for chloroplast function, providing a platform for the photosynthetic protein pigment complexes and the conversion of energy throughout the process of photosynthesis $[38,39]$. We performed TEM in our study and discovered a strong contrast between the chloroplast ultrastructure of yellow and green leaves. The ultrastructure of mutant chloroplasts had serious defects, with some chloroplasts containing broken thylakoid membranes packed with vesicles and filled with a large number of plastid spheres. Furthermore, the chloroplast number in the mutant leaves decreased in comparison to wild type green leaves. In contrast, the structures in the chloroplasts of green leaves were intact, clearly visible, and well-organized. This result indicates that the yellow leaf phenotype might be a consequence of disordered chloroplast development. Similar results were also reported in the green leaves of Ginkgo biloba [6], rice [39], Pseudosasa japonica [40], Ilex $\times$ altaclerensis [41] and Anthurium andraeanum [42].

Transcriptomic analysis has been widely used to identify key genes that are differentially expressed at different developmental stages, or under various physiological conditions. However, the genome of Paphiopedilum is still relatively unknown. In this study, RNA-sequencing analysis on P. SCBG COP15 leaf phenotypes (Y and G) at the same development stage were sequenced and annotated. Finally, we obtained 113,567 transcripts with N50 lengths of $1800 \mathrm{bp}$, and 79,129 unigenes with N50 lengths of 1590 $\mathrm{bp}$, similar to those in other orchid species, such as Cymbidium longibracteatum [35] and A. shenzhenica [43], respectively. Further, a total of 1835 DEGs were identified by TPM, with 463 DEGs successfully assigned to 111 KEGG pathways. The most frequently represented pathway was the plant hormone signal transduction. The results suggested that plant hormone is playing an important role in the formation of plants leaf yellowing, which has also been reported in the Arabidopsis genus [44-46].

Chl biosynthesis is catalyzed by 15 kinds of enzymes, and any disturbance in this process results in Chl deficiency and leaf color mutation [47]. HEMA is the first structural gene in Chl biosynthesis, encoding Glutamyl-tRNA reductase which catalyzes the initial substrate glutamyl-tRNA to glutamate-1-semialdehyde (GSA), with the unstable intermediate GSA then being isomerized to 5-aminolevulinic acid by GSA aminotransferase [48]. Previous research has shown that the mutation of the HEMA gene in rice causes the entire Chl synthesis pathway to be blocked, with the apparent yellow plant leaves visible [49], and RNA silencing of HEMA in barley showed a similar phenotype [8]. In Arabidopsis, HEMA1 mutants are patchy to completely yellow and cannot grow healthily under normal growth conditions [50]. From our transcriptomic analysis, a HEMA (TRINITY_DN6358_c0_g1) gene was identified as a critical structural gene responsible for yellow mutant formation, due to its decreased expression level. In addition, CRD (TRINITY_DN2556_c0_g1), 
CAO (TRINITY_DN1555_c0_g1), and CHLE (TRINITY_DN2556_c0_g1) also showed low mRNA levels in yellow leaves. In contrast, the Chl degradation genes NYC1-1 (TRINITY_DN221_c0_g1), PPH (TRINITY_DN16260_c0_g1 and TRINITY_DN3203_c0_g1), and HCAR (TRINITY_DN482_c0_g1) had significantly increased expression levels in the yellow leaves, which indicates that the upregulated degradation genes in the mutant may accelerate $\mathrm{Chl}$ breakdown and lead to the yellowing of plant leaves. Specific expression levels of TPM related to Chl biosynthesis and degradation are provided in Supplementary Table S8.

\section{Conclusions}

We performed comparative analysis to investigate the differences in coloration between normal green leaves and yellow mutants of a Paphiopedilum hybrid. The low $\mathrm{Chl}$ content and abnormal ultrastructure of chloroplasts in the leaves of the yellow mutant suggested that $\mathrm{Chl}$ biosynthesis was partially inhibited, which could explain the yellow phenotype from both cytological and physiological aspects. Key structural genes related to $\mathrm{Chl}$ biosynthesis and degradation, along with potential transcription factors, were identified by DEG analysis from transcriptomic data. In summary, from our cytological, physiological, and transcriptomic analyses, decreasing the amount of photosynthetic pigments, blocking chloroplast development, and changing the expression levels of genes involved in Chl biosynthesis and degradation may collectively lead to the yellow leaf phenotype (Figure 10). Our findings provide an essential genetic resource, not only for the study of molecular mechanisms involved in leaf color variation, but also for the breeding of new varieties of Paphiopedilum.
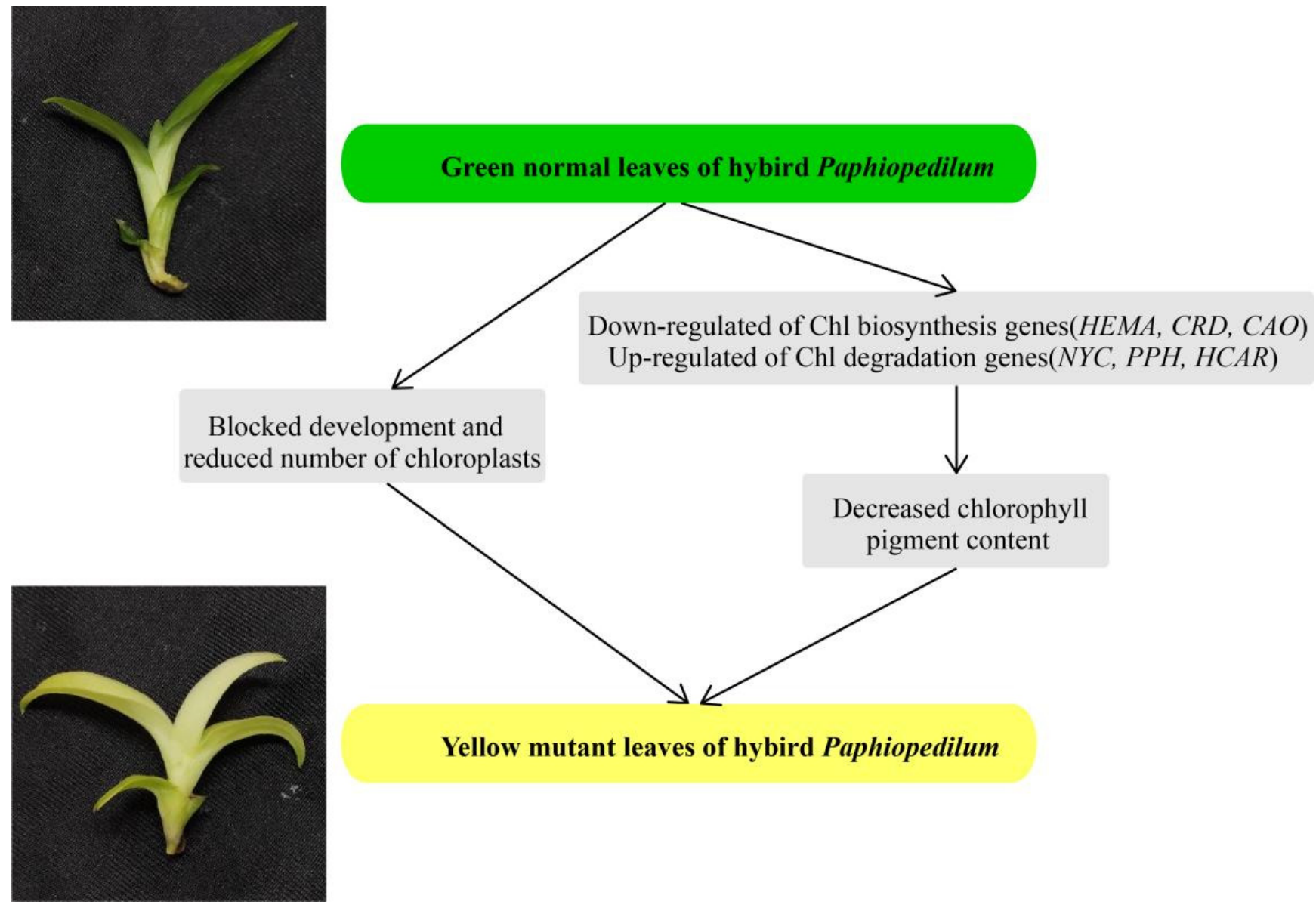

Figure 10. A proposed model for the mutant leaf coloration in the Paphiopedilum hybrid. 
Supplementary Materials: The following supporting information can be downloaded at: https: / / www.mdpi.com/article/10.3390/genes13010071/s1. Table S1: A list of the plant environment, Table S2: A summary of the quality of the transcriptome, Table S3: A summary of the assemble results, Table S4: Overview of transcriptome assembly showing length distribution of unigenes, Table S5: Identification of coding sequences (CDS) and protein sequences of Chl biosynthesis and degradation. Gene sequences were screened by RNA-Seq date and confirmed by PCR; Table S6: TPM value of 20 DEGs which exhibited highly significant down or up-regulation between yellow and green leaves, Table S7: Primers used in quantitative real-time polymerase chain reaction (qRT-PCR), Table S8: Specific expression levels of transcripts per million reads (TPM) value related to Chl biosynthesis and degradation, Figure S1: Schematic representation of Chl metabolic pathways in higher plants, Figure S2: The quality of RNA used for qPCR assessed by agarose gel electrophoresis. 1-4: yellow leaves, 5-8: green leaves, Figure S3: Correlation indices between different samples. Y1-Y4, yellow leaves; G1-G4, green leaves, Figure S4: Species distribution of NR annotation. The most abundant annotated species are D. catenatum (15,014 unigenes, $44.21 \%)$, Phalaenopsis equestris (3407 unigenes, $10.03 \%$ ) and Apostasia shenzhenica (3320 unigenes, 9.78\%), which all belong to the orchid family, Figure S5: GO functional annotation of all unigenes. The most abundant functions in terms of biological processes are cellular processes and metabolic processes. In terms of cellular components, the most abundant unigenes are related to cell parts and membrane parts, and in terms of molecular function the most abundant unigenes are related to binding and catalytic activity, Figure S6: KEGG annotation of all unigenes.

Author Contributions: J.L., L.F. and S.Z. conceived, designed, and assessed the experiments; J.L., K.W., M.W. and L.L. performed the experiments; J.L., K.W., L.F. and S.Z. wrote the manuscript. All authors reviewed the manuscript, approved all edits in the final version and take public responsibility for the content. All authors have read and agreed to the published version of the manuscript.

Funding: This work was funded by the National Key Research and Development Program of China (2018YFD1000403), the National Natural Science Foundation of China (31902062), the Natural Science Foundation of Guangdong Province (2018A030313686), the Guangdong Key Technology Research and Development Program (2018B020202001-3), the Guangdong Modern Agricultural Industry Technology System Program (2021KJ121), and the Shenzhen Key Laboratory for Orchid Conservation and Utilization (OU202101).

Institutional Review Board Statement: The study was conducted according to the guidelines of the Declaration of Helsinki, and approved by the Institutional Review Board of South China Botanical Garden, Chinese Academy of Sciences.

Informed Consent Statement: There were no human or animal research subjects in this study. Consequently, informed consent was not required.

Data Availability Statement: Data may be found within the article or supplementary material. Raw data available upon reasonable request.

Conflicts of Interest: The authors declare no conflict of interest.

\section{Abbreviations}

HEMA: glutamyl-tRNA reductase; HEML: glutamate-1-semialdehyde 2,1-aminotransferase; HEMB: 5-aminolevulinate dehydrogenase; HEMC: porphobilinogen deaminase; HEMD: uroporphyrinogen III synthase; HEME: uroporphyrinogen III decarboxylase; HEMF: coproporphyrinogen III oxidase; HEMG: protoporphyrinogen oxidase; CHLH: Mg-chelatase; Mgprotoporphyrin IX methyltransferase; CHLM: Mg-protoporphyrin IX methyltransferase; CRD: Mg-protoporphyrin IX monomethylester cyclase; PORA/B/C: protochlorophyllide oxidoreductase; DVR: divinyl chlorophyllide a 8-vinyl-reductase; CHLG: Chl synthase; CAO: chlorophyllide a oxygenase; NYC1: Chl b reductase; HCAR: hydroxymethyl Chl a reductase; SGR: STAY-GREEN (Mg-dechelatase); PPH: pheophytinase; PaO: pheophorbide a oxygenase; RCCR: red Chl catabolite reductase. 


\section{References}

1. Esteban, R.; Fernandez-Marin, B.; Becerril, J.M.; Garcia-Plazaola, J.I. Photoprotective implications of leaf variegation in E-denscanis L. and P-officinalis L. J. Plant Physiol. 2008, 165, 1255-1263. [CrossRef] [PubMed]

2. Eckhardt, U.; Grimm, B.; Hortensteiner, S. Recent advances in chlorophyll biosynthesis and breakdown in higher plants. Plant Mol. Biol. 2004, 56, 1-14. [CrossRef] [PubMed]

3. Feild, T.S.; Lee, D.W.; Holbrook, N.M. Why leaves turn red in autumn. The role of anthocyanins in senescing leaves of red-osier dogwood. Plant Physiol. 2001, 127, 566-574. [CrossRef] [PubMed]

4. Nie, L.B.; Zheng, Y.S.; Zhang, L.T.; Wu, Y.; Zhu, S.D.; Hou, J.F.; Chen, G.H.; Tang, X.Y.; Wang, C.G.; Yuan, L.Y. Characterization and transcriptomic analysis of a novel yellow-green leaf wucai (Brassica campestris L.) germplasm. BMC Genom. 2021, 22, 258. [CrossRef] [PubMed]

5. Chang, Q.S.; Zhang, L.X.; Hou, X.G.; Wang, Z.; Wang, N.; Gong, M.G.; Zhang, Q.M.; Chen, H.; Shi, Z.Q.; Deng, C.C. The anatomical, physiological, and molecular analysis of a chlorophyll-deficient mutant in tree peony (Paeonia suffruticosa). Photosynthetica 2019, 57, 724-730. [CrossRef]

6. $\quad$ Li, W.X.; Yang, S.B.; Lu, Z.G.; He, Z.C.; Ye, Y.L.; Zhao, B.B.; Wang, L.; Jin, B. Cytological, physiological, and transcriptomic analyses of golden leaf coloration in Ginkgo biloba L. Hortic Res. Engl. 2018, 5, 12. [CrossRef] [PubMed]

7. Nagata, N.; Tanaka, R.; Satoh, S.; Tanaka, A. Identification of a vinyl reductase gene for chlorophyll synthesis in Arabiopsis thalinana. Plant Cell Physiol. 2005, 46, S89.

8. Petersen, B.L.; Moller, M.G.; Jensen, P.E.; Henningsen, K.W. Identification of the Xan-g gene and expression of the Mg-chelatase encoding genes Xan-f, -g and -h in mutant and wild type barley (Hordeum vulgare L.). Hereditas 1999, 131, 165-170. [CrossRef]

9. Zhang, H.T.; Li, J.J.; Yoo, J.H.; Yoo, S.C.; Cho, S.H.; Koh, H.J.; Seo, H.S.; Paek, N.C. Rice chlorina-1 and chlorina-9 encode ChlD and ChlI subunits of Mg-chelatase, a key enzyme for chlorophyll synthesis and chloroplast development. Plant Mol. Biol. 2006, 62, 325-337. [CrossRef] [PubMed]

10. Hortensteiner, S. Stay-green regulates chlorophyll and chlorophyll-binding protein degradation during senescence. Trends Plant Sci. 2009, 14, 155-162. [CrossRef]

11. Sato, Y.; Morita, R.; Nishimura, M.; Yamaguchi, H.; Kusaba, M. Mendel's green cotyledon gene encodes a positive regulator of the chlorophyll-degrading pathway. Proc. Natl. Acad. Sci. USA 2007, 104, 14169-14174. [CrossRef] [PubMed]

12. Ren, G.D.; An, K.; Liao, Y.; Zhou, X.; Cao, Y.J.; Zhao, H.F.; Ge, X.C.; Kuai, B.K. Identification of a novel chloroplast protein AtNYE1 regulating chlorophyll degradation during leaf senescence in Arabidopsis. Plant Physiol. 2007, 144, 1429-1441. [CrossRef] [PubMed]

13. Fang, L.; Xu, X.; Li, J.; Zheng, F.; Li, M.Z.; Yan, J.W.; Li, Y.; Zhang, X.H.; Li, L.; Ma, G.H.; et al. Transcriptome analysis provides insights into the non-methylated lignin synthesis in Paphiopedilum armeniacum seed. BMC Genom. 2020, 21, 524. [CrossRef] [PubMed]

14. Yukawa, T.; Stern, W.L. Comparative vegetative anatomy and systematics of Cymbidium (Cymbidieae: Orchidaceae). Bot. J. Linn Soc. 2002, 138, 383-419. [CrossRef]

15. Li, Y.H.; Imai, K.; Ohno, H.; Matsui, S. Effects of acclimatization temperatures on antioxidant enzyme activities in mericlones of a cattleya hybrid. J. Jpn. Soc. Hortic. Sci. 2004, 73, 386-392. [CrossRef]

16. Zhang, J.X.; Wu, K.L.; Zeng, S.J.; Teixeira da Silva, J.A.; Zhao, X.L.; Tian, C.E.; Xia, H.Q.; Duan, J. Transcriptome analysis of Cymbidium sinense and its application to the identification of genes associated with floral development. BMC Genom. 2013, 14, S1. [CrossRef]

17. Chugh, S.; Guha, S.; Rao, I.U. Micropropagation of orchids: A review on the potential of different explants. Sci Hortic-Amst. 2009, 122, 507-520. [CrossRef]

18. Leitch, I.J.; Kahandawala, I.; Suda, J.; Hanson, L.; Ingrouille, M.J.; Chase, M.W.; Fay, M.F. Genome size diversity in orchids: Consequences and evolution. Ann. Bot. Lond. 2009, 104, 469-481. [CrossRef]

19. Zeng, S.J.; Huang, W.C.; Wu, K.L.; Zhang, J.X.; Teixeira da Silva, J.A.; Duan, J. In vitro propagation of Paphiopedilum orchids. Crit. Rev. Biotechnol. 2016, 36, 521-534. [CrossRef]

20. Lichtenthaler, H.K. Chlorophylls and carotenoids-Pigments of photosynthetic biomembranes. Method Enzymol. 1987, 148, 350-382.

21. Conesa, A.; Gotz, S.; Garcia-Gomez, J.M.; Terol, J.; Talon, M.; Robles, M. Blast2GO: A universal tool for annotation, visualization and analysis in functional genomics research. Bioinformatics 2005, 21, 3674-3676. [CrossRef] [PubMed]

22. Kanehisa, M.; Goto, S. KEGG: Kyoto encyclopedia of genes and genomes. Nucleic Acids Res. 2000, 28, 27-30. [CrossRef] [PubMed]

23. Love, M.I.; Huber, W.; Anders, S. Moderated estimation of fold change and dispersion for RNA-seq data with DESeq2. Genome Biol. 2014, 15, 550. [CrossRef] [PubMed]

24. Xie, C.; Mao, X.Z.; Huang, J.J.; Ding, Y.; Wu, J.M.; Dong, S.; Kong, L.; Gao, G.; Li, C.Y.; Wei, L.P. KOBAS 2.0: A web server for annotation and identification of enriched pathways and diseases. Nucleic Acids Res. 2011, 39, W316-W322. [CrossRef]

25. Livak, K.J.; Schmittgen, T.D. Analysis of relative gene expression data using real-time quantitative PCR and the 2(T)(-Delta Delta C) method. Methods 2001, 25, 402-408. [CrossRef]

26. Hall, L.N.; Rossini, L.; Cribb, L.; Langdale, J.A. GOLDEN 2: A novel transcriptional regulator of cellular differentiation in the maize leaf. Plant Cell 1998, 10, 925-936. [CrossRef] 
27. Fitter, D.W.; Martin, D.J.; Copley, M.J.; Scotland, R.W.; Langdale, J.A. GLK gene pairs regulate chloroplast development in diverse plant species. Plant J. 2002, 31, 713-727. [CrossRef]

28. Waters, M.T.; Wang, P.; Korkaric, M.; Capper, R.G.; Saunders, N.J.; Langdale, J.A. GLK Transcription factors coordinate expression of the photosynthetic apparatus in Arabidopsis. Plant Cell 2009, 21, 1109-1128. [CrossRef]

29. Zeng, S.J.; Wu, K.L.; Teixeira da Silva, J.A.; Zhang, J.X.; Chen, Z.L.; Xia, N.H.; Duan, J. Asymbiotic seed germination, seedling development and reintroduction of Paphiopedilum wardii Sumerh., an endangered terrestrial orchid. Sci. Hortic. Amst. 2012, 138, 198-209. [CrossRef]

30. Li, X.; Kanakala, S.; He, Y.H.; Zhong, X.L.; Yu, S.M.; Li, R.X.; Sun, L.X.; Ma, J. Physiological characterization and comparative transcriptome analysis of white and green leaves of Ananas comosus var. bracteatus. PLoS ONE 2017, 12, e0169838. [CrossRef]

31. Larkin, P.J.; Scowcroft, W.R. Somaclonal variation-A novel source of variability from cell-cultures for plant improvement. Theor. Appl. Genet. 1981, 60, 197-214. [CrossRef] [PubMed]

32. Broughton, S. Ovary co-culture improves embryo and green plant production in anther culture of Australian spring wheat (Triticum aestivum L.). Plant Cell Tiss Org. 2008, 95, 185-195. [CrossRef]

33. Martin, C.; Uberhuaga, E.; Perez, C. Application of RAPD markers in the characterisation of Chrysanthemum varieties and the assessment of somaclonal variation. Euphytica 2002, 127, 247-253. [CrossRef]

34. Sato, M.; Kawabe, T.; Hosokawa, M.; Tatsuzawa, F.; Doi, M. Tissue culture-induced flower-color changes in Saintpaulia caused by excision of the transposon inserted in the flavonoid $3^{\prime}, 5^{\prime}$ hydroxylase (F3' $5^{\prime} \mathrm{H}$ ) promoter. Plant Cell Rep. 2011, 30, 929-939. [CrossRef] [PubMed]

35. Yu, J.; Qiang, W.; Qin-Qin, S.; Bi-Ping, Z.; Jun-Rong, H. Transcriptome analysis reveals genes associated with leaf color mutants in Cymbidium longibracteatum. Tree Genet. Genomes 2020, 16, 44. [CrossRef]

36. Lonosky, P.M.; Zhang, X.S.; Honavar, V.G.; Dobbs, D.L.; Fu, A.; Rodermel, S.R. A proteomic analysis of maize chloroplast biogenesis. Plant Physiol 2004, 134, 560-574. [CrossRef] [PubMed]

37. Maekawa, S.; Takabayashi, A.; Reyes, T.H.; Yamamoto, H.; Tanaka, A.; Sato, T.; Yamaguchi, J. Pale-green phenotype of atl31 atl6 double mutant leaves is caused by disruption of 5-aminolevulinic acid biosynthesis in Arabidopsis thaliana. PLoS ONE 2015, 10, e0117662. [CrossRef]

38. Benning, C. A role for lipid trafficking in chloroplast biogenesis. Prog Lipid Res. 2008, 47, 381-389. [CrossRef] [PubMed]

39. Wu, Z.M.; Zhang, X.; Wang, J.L.; Wan, J.M. Leaf chloroplast ultrastructure and photosynthetic properties of a chlorophyll-deficient mutant of rice. Photosynthetica 2014, 52, 217-222. [CrossRef]

40. Yang, H.Y.; Xia, X.W.; Fang, W.; Fu, Y.; An, M.M.; Zhou, M.B. Identification of genes involved in spontaneous leaf color variation in Pseudosasa japonica. Genet Mol. Res. 2015, 14, 11827-11840. [CrossRef]

41. Zhang, Q.; Huang, J.; Zhou, P.; Hao, M.; Zhang, M. Cytological and transcriptomic analysis provide insights into the formation of variegated leaves in Ilex $\times$ altaclerensis 'Belgica Aurea'. Plants 2021, 10, 552. [CrossRef]

42. Yang, Y.X.; Chen, X.X.; Xu, B.; Li, Y.X.; Ma, Y.H.; Wang, G.G. Phenotype and transcriptome analysis reveals chloroplast development and pigment biosynthesis together influenced the leaf color formation in mutants of Anthurium andraeanum 'Sonate'. Front. Plant Sci. 2015, 6, 139. [CrossRef]

43. Zhang, L.S.; Chen, F.; Zhang, G.Q.; Zhang, Y.Q.; Niu, S.; Xiong, J.S.; Lin, Z.G.; Cheng, Z.M.; Liu, Z.J. Origin and mechanism of crassulacean acid metabolism in orchids as implied by comparative transcriptomics and genomics of the carbon fixation pathway. Plant J. 2016, 86, 175-185. [CrossRef] [PubMed]

44. Qi, T.C.; Wang, J.J.; Huang, H.; Liu, B.; Gao, H.; Liu, Y.L.; Song, S.S.; Xie, D.X. Regulation of jasmonate-induced leaf senescence by antagonism between bHLH subgroup IIIe and IIId factors in Arabidopsis. Plant Cell 2015, 27, 1634-1649. [CrossRef] [PubMed]

45. Guo, Y.F.; Gan, S.S. AtNAP, a NAC family transcription factor, has an important role in leaf senescence. Plant J. 2006, 46, 601-612. [CrossRef] [PubMed]

46. Reymond, P.; Farmer, E.E. Jasmonate and salicylate as global signals for defense gene expression. Curr. Opin. Plant Biol. 1998, 1 , 404-411. [CrossRef]

47. Zhao, M.H.; Li, X.; Zhang, X.X.; Zhang, H.; Zhao, X.Y. Mutation mechanism of leaf color in plants: A Review. Forests 2020, 11, 851. [CrossRef]

48. Terry, M.J.; Smith, A.G. A model for tetrapyrrole synthesis as the primary mechanism for plastid-to-nucleus signaling during chloroplast biogenesis. Front. Plant Sci. 2013, 4, 14. [CrossRef]

49. Liu, W.Z.; Fu, Y.P.; Hu, G.C.; Si, H.M.; Zhu, L.; Wu, C.; Sun, Z.X. Identification and fine mapping of a thermo-sensitive chlorophyll deficient mutant in rice (Oryza sativa L.). Planta 2007, 226, 785-795. [CrossRef]

50. Kumar, A.M.; Soll, D. Antisense HEMA1 RNA expression inhibits heme and chlorophyll biosynthesis in Arabidopsis. Plant Physiol. 2000, 122, 49-55. [CrossRef] 